\title{
Efeitos de um programa de caminhada sob os níveis de autonomia funcional de idosas monitoradas pelo programa saúde da família
}

Effects of the research of hiking related the profiles of functional autonomy in elderly women supported by the family health program

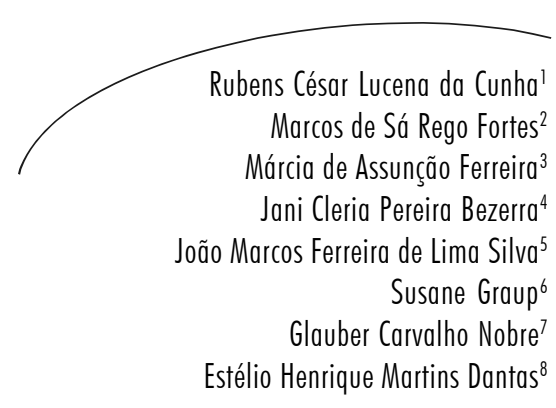

Resumo

Objetivo: verificar os efeitos de um programa de caminhada sobre os níveis de autonomia funcional de idosas participantes do Programa Saúde da Família. Métodos: Para este estudo experimental, a amostra foi aleatoriamente dividida em: grupo experimental (GE; $n=58$, idade: $67 \pm 6$ anos) e grupo controle (GC; $n=40$; idade: $70 \pm 6$ anos). Todas eram fisicamente ativas, porém sem um rigor na regularidade do programa de atividade física. A autonomia funcional foi avaliada pela bateria de testes do Grupo de Desenvolvimento Latino-Americano para a Maturidade (GDLAM), composta de: caminhar $10 \mathrm{~m}(\mathrm{C} 10 \mathrm{~m})$, levantar-se da posição sentada (LPS), levantar-se da posição de decúbito ventral (LPDV), levantar-se da cadeira e locomover-se pela a casa (LCLC) e o teste de vestir e tirar uma camiseta (VTC). Esses testes resultam no índice de GDLAM (IG). O programa de intervenção foi realizado em frequência semanal de três vezes, com intensidade que variava entre $55 \%$ a $75 \%$ da frequência cardíaca máxima. Resultados: $\mathrm{Na}$ análise intragrupos, pôdese observar melhora significativa no GE, em todos os testes do GDLAM e no IG $(\Delta=-4,13 ; \mathrm{p}=0,0001)$; entretanto, o GC obteve uma piora significativa em todos os testes do GDLAM e no IG $(\Delta=+0,05 ; \mathrm{p}=0,0001)$. Na comparação intergrupos, o GE mostrou-se superior ao GC em todos os testes $(\mathrm{p}<0,05)$ e no IG $(\Delta=-8,23 ; \mathrm{p}=0,0001)$. Conclusões: Pode-se inferir que o GE, após ter se submetido à atividade de caminhada regular e controlada, obteve melhora do nível de autonomia funcional, quando comparado ao inicial e ao do GC. Número de registro clínico: NCT00981734.

\footnotetext{
Universidade Castelo Branco, Fisiologia do Exercício. Rio de Janeiro, RJ, Brasil.

2 Instituto de Pesquisa da Capacitação Física do Exército. Forte São João. Rio de Janeiro, RJ, Brasil.

3 Universidade Federal do Rio de Janeiro, Escola de Enfermagem Anna Nery. Rio de Janeiro, RJ, Brasil.

4 Universidade Castelo Branco, Motricidade Humana. Rio de Janeiro, RJ, Brasil.

5 Instituto Federal de Educação, Ciência e Tecnologia do Ceará, Campus Juazeiro do Norte. Juazeiro do Norte, CE, Brasil.

6 Universidade Federal de Santa Catarina, Laboratório de Biomecânica. Florianópolis, SC, Brasil.

7 Universidade do Estado de Santa Catarina. Pós-graduação em Ciências do Movimento Humano. São José, SC, Brasil

8 Universidade Castelo Branco, Faculdade de Educação e Desporto. Rio de Janeiro, RJ, Brasil.
}

\author{
Palavras-chave: \\ Caminhada. Autonomia \\ Pessoal. Idoso. Mulheres. \\ Programa Saúde da \\ Família. Envelhecimento. \\ Estilo de vida. \\ Funcionalidade.
}




\section{Abstract}

Objective: To evaluate the effects of a walking program on levels of functional autonomy of elderly participants of the Family Health Program. Methods: In this experimental study, the sample was randomly divided into: experimental group (EG, $\mathrm{n}=58$, age: $67 \pm 6$ years) and a control group ( $n=40$, age: $70 \pm 6$ years). All were physically active but without a rigorous program of regular physical activity. Functional autonomy was evaluated by the battery of tests of the Group of Latin American Development to Maturity (GDLAM), comprising: walk $10 \mathrm{~m}(\mathrm{C} 10 \mathrm{M})$, rising from a seated position (LPS), rising from the prone position (LPDV), rising from his chair and moving around the house (LCLC) and test and get a dress shirt (VTC). These tests result in GDLAM index (GI). The intervention program was conducted in weekly frequency of three times, with intensity ranging from $55 \%$ to $75 \%$ of maximum heart rate. Results: In the intragroup analysis, one could observe a significant improvement in the SG in all tests GDLAM and IG $(\Delta=-4.13, \mathrm{p}=0.0001)$, although the GC obtained a significant worsening in all GDLAM tests and IG $(\Delta=+0.05, \mathrm{p}=0.0001)$. In intergroup comparison, the GE was superior to GC in all tests $(p<0.05)$ and IG $(\Delta=-8.23$, $\mathrm{p}=0.0001)$. Conclusions: It can be inferred that the $\mathrm{GE}$, after undergoing regular walking activity and controlled, had improved the level of functional autonomy, compared to the initial and the GC. Number of clinical register: NCT00981734
Key Words: Walking. Personal Autonomy. Aged. Women. Family Health Program. Aging. Life Style. Functionality.

\section{INTRODUÇ̃̃̃O}

O crescimento da população idosa vem acontecendo de forma progressiva no Brasil. Segundo dados do Instituto Brasileiro de Geografia e Estatística (IBGE), os valores da projeção dessa população seguem uma curva de crescimento acelerado onde o Brasil será o sexto país do mundo em número de idosos até 2025. Já no Estado do Ceará, existem 607.030 idosos, dos quais 359.624 são mulheres de acordo com o Censo 2000, o que corresponde a $4,5 \%$ das 8.002 .245 de idosas existentes em todo o Brasil. ${ }^{1}$

O processo de envelhecimento é acompanhado por uma diminuição da capacidade funcional que está associada ao declínio na habilidade para desempenhar as atividades da vida diária (AVD). Pode ser a maior razão para a perda da autonomia e da independência dos idosos, tornando as pessoas dependentes de outras, resultado de um estilo de vida sedentário vivido ao longo dos anos. ${ }^{2}$

As alterações biológicas, tais como perda óssea, perda de massa magra, concentração de tecido adiposo, mudanças psicossociais (entre as quais diminuição da sociabilidade, mudanças no controle emocional, baixa autoestima), vivenciadas com o passar do tempo, são determinadas pelo estilo de vida adotado na velhice e nas idades pregressas. ${ }^{3}$ Se o idoso pratica e praticou atividade física durante toda a sua vida, essas alterações serão minimizadas e a autonomia funcional será mantida. ${ }^{4}$

Dentre as atividades mais verificadas em programas de exercícios físicos para idosos, podese destacar a caminhada, que contribui para uma melhora na circulação e na atividade do coração, além de diminuir os riscos de problemas cardíacos. $^{5}$

$\mathrm{Na}$ caminhada, podemos encontrar um exemplo de atividade física ideal para idosos, pois não implica riscos à saúde, tem sua intensidade controlada facilmente, não exige revisão médica para quem é saudável, além de não apresentar grandes custos para sua realização. Não obstante, são associados a esta prática inúmeros benefícios à saúde, podendo ainda ser realizada em grupo, embora os limites físicos sejam respeitados individualmente. $^{6}$

Sendo assim, o objetivo deste estudo foi verificar os efeitos de um programa de caminhada sobre os níveis de autonomia funcional de idosas pertencentes ao Programa Saúde da Família (PSF). 


\section{METODOLOGIA}

\section{Amostra}

O presente estudo buscou investigar mulheres idosas cadastradas no PSF localizado na cidade de Juazeiro do Norte, região sul do Estado do Ceará. Inicialmente, foi realizado levantamento do número de idosas cadastradas no PSF. No período em que a pesquisa foi realizada, 200 idosas compareceram no PSF, sendo convidadas a participar do estudo e submetidas a uma entrevista, visando a identificar as condições estabelecidas pelos critérios de inclusão e exclusão.

Como critérios de inclusão, adotou-se: apresentar idade igual ou superior a 60 anos e ser acompanhada periodicamente pelo PSF. Os critérios de exclusão foram: o acometimento por enfermidades agudas ou crônicas que pudessem comprometer ou se tornar um fator de impedimento para a realização das avaliações e o uso de medicamentos que causassem distúrbios de atenção.

Após os critérios de inclusão e exclusão, a amostra foi composta por 98 idosas, divididas de forma probabilística (por sorteio) em: grupo experimental (GE; $\mathrm{n}=58$, idade: $67 \pm 6$ anos) e grupo controle (GC; $n=40$; idade: $70 \pm 6$ anos).

O estudo atendeu às normas determinadas pela Declaração de Helsinki para pesquisas envolvendo seres humanos, sendo aprovada sua realização pelo Comitê de Ética em Pesquisa da Universidade Castelo Branco em 19/07/2008, sob o protocolo de número 0042/2008. ${ }^{7}$

\section{Procedimentos}

Para a avaliação física, solicitou-se às participantes que utilizassem traje de banho (maio) para mensuração das medidas de massa corporal, estatura e IMC. Foi realizado o cadastro de todas as participantes com acompanhamento sistemático através de avaliação física e clínica durante 16 semanas.

A primeira coleta de dados aconteceu na primeira semana do mês de abril e a segunda, na primeira semana do mês de agosto de 2009, ambas no turno da manhã.

Para avaliar a massa corporal, foi utilizada uma Balança da Filizola com selo do INMETRO (precisão de $100 \mathrm{~g}$ ). A estatura foi mensurada através de um estadiômetro com selo do INMETRO (precisão de $1 \mathrm{~mm}$ ). A partir dessas medidas, o índice de massa corporal (IMC) foi calculado, mais precisamente dividindo-se o peso em quilogramas pelo quadrado da estatura em metros. Com base no IMC, considerou-se como baixo-peso $\mathrm{IMC}<18,5 \mathrm{~kg} / \mathrm{m} 2$, sobrepeso IMC $\geq 25 \mathrm{~kg} / \mathrm{m} 2$ e obesidade IMC $>30 \mathrm{~kg} / \mathrm{m} 2$.

Para a avaliação da autonomia funcional, foi aplicada a bateria do Grupo de Desenvolvimento Latino-Americano para a Maturidade (GDLAM), proposta por Dantas \& Vale, ${ }^{8}$ composta por: caminhada de 10 metros $(\mathrm{C} 10 \mathrm{~m}) ;$ levantar-se da posição sentada (LPS); ${ }^{10}$ levantarse da posição de decúbito ventral (LPDV); ${ }^{11}$ levantar-se da cadeira e locomover-se pela casa (LCLC) ${ }^{12}$ e o teste de vestir e tirar uma camiseta (VTC), ${ }^{13}$ todos medidos em segundos, os quais integram de forma ponderada um escore chamado de Índice Geral do GDLAM (IG). ${ }^{14}$

Os valores de referência para classificação do nível de autonomia funcional, considerando o IG e seus componentes, foram os próprios sugeridos pelo GDLAM..$^{14}$ (Tabela 1 ) 
Tabela 1 - Padrões de avaliação da autonomia funcional do protocolo GDLAM. Juazeiro do Norte, CE, 2009.

\begin{tabular}{lllllll}
\hline Classificação & C10M $^{1}$ & LPS & LPDV & VTC & LCLC & IG $^{1}$ \\
\hline Fraco & $+7,09$ & $+11,19$ & $+4,40$ & $+13,14$ & $+43,00$ & $+27,42$ \\
Regular & $7,09-6,34$ & $11,19-9,55$ & $4,40-3,30$ & $13,14-11,62$ & $43,00-38,69$ & $27,42-24,98$ \\
Bom & $6,33-5,71$ & $9,54-7,89$ & $3,29-2,63$ & $11,61-10,14$ & $38,68-34,78$ & $24,97-22,66$ \\
Muito bom & $-5,71$ & $-7,89$ & $-2,63$ & $-10,14$ & $-34,78$ & $-22,66$ \\
\hline
\end{tabular}

C10M - caminhar 10m; LPS - levantar-se da posição sentada; LPDV - Levantar-se da posição decúbito ventral; LCLC - Levantar-se da cadeira e locomover-se pela casa; VTC - Vestir e tirar uma camiseta; IG: Índice GDLAM / ${ }^{1}$ Segundos / ${ }^{2}$ Escores

O programa de intervenção foi realizado em frequência semanal de três vezes (segundas, quartas e sextas-feiras) nos horários das $17 \mathrm{~h}$ às $17 \mathrm{~h} 45 \mathrm{~min}$, com intensidade que variava entre $55 \%$ a $75 \%$ da frequência cardíaca máxima (FCM) obtida pela equação: $\mathrm{FCM}=220$-idade. A FCM foi controlada pela aferição da frequência cardíaca (FC) manual na carótida em seis segundos e multiplicada por 10. Esta FC era mensurada pelas próprias participantes, as quais receberam treinamento $\mathrm{e}$ orientações para que a fizesse de forma correta.

Todas as participantes tinham sua FC aferida por um dos monitores, pelo menos uma vez por sessão, logo após a autoaferição, a fim de confirmar o valor identificado pela idosa. $\mathrm{O}$ momento de aferição da FC era variado, sendo realizada a partir do comando do professor responsável ou solicitada pelo monitor próximo à idosa. forma:

As sessões foram distribuídas da seguinte

- Aquecimento: duração 5 a 10 minutos de exercícios articulares dos membros superiores, pescoço, tronco e membros inferiores.

- Propriamente dita: 20 a 30 minutos de caminhada no método natural.

- Volta à calma: 3 a 5 minutos de alongamentos sentado e/ou deitados dos segmentos: pescoço, membros superiores, tronco e membros inferiores. Utilizando o método alternado por segmento. A atividade de intervenção foi orientada em ambiente controlado quanto à superfície (plana e não escorregadia) e marcação das distâncias percorridas (pista de atletismo não oficial - 260 metros), onde o treinamento foi controlado em função do tempo de caminhada, conforme diretrizes do American College Sport of Medicine ${ }^{15}$ e da $5^{\text {a }}$ Diretriz Brasileira de Hipertensão Arterial, ${ }^{16}$ que preconizam uma frequência mínima de três vezes por semana com duração entre 20 e 30 minutos como adequados para que sejam significativas as adaptações fisiológicas neste grupo etário.

\section{Tratamento Estatístico}

Foi utilizada estatística descritiva com média, erro-padrão, mediana, desvio-padrão, mínimo, máximo e delta absoluto. A normalidade da amostra foi avaliada pelo teste de Shapiro-Wilk (GC) e de Kolmogorov Smirnov (GE). Para a análise das variáveis respostas, foi utilizado na análise intragrupos, o teste t-Student pareado ou de Wilcoxon, quando apropriado (distribuição homogênea ou heterogênea dos dados, respectivamente). Para a avaliação intergrupos, foi utilizado o teste paramétrico de ANOVA two way, seguido do Post Hoc de Scheffe. Adotou-se o nível de $\mathrm{p}<0.05$ para a significância estatística. Para a avaliação dos resultados, foram utilizados o programa Excell e o Statistical Package for the Social Science ${ }^{\circledR}$ (SPSS), versão 14.0. 
RESULTADOS

A análise da tabela 2, referente às características antropométricas do GE e GC, permite identificar que os valores das médias de IMC dos dois grupos indicam estado nutricional de sobrepeso tanto no pré quanto no pós-teste. No entanto, a média do IMC diminuiu no GE após o período de intervenção, o que não foi observado no GC. Cabe ressaltar que a estatura no GE e o IMC no GC não apresentaram distribuição normal, sendo essas variáveis avaliadas, posteriormente, por testes não-paramétricos.

Tabela 2 - Análise descritiva dos dados antropométricos da amostra. Juazeiro do Norte, CE, 2009.

\begin{tabular}{|c|c|c|c|c|c|c|c|c|c|}
\hline & & & Média & $\begin{array}{l}\text { Erro- } \\
\text { padrão }\end{array}$ & Mediana & $\begin{array}{l}\text { Desvio- } \\
\text { padrão }\end{array}$ & Mínimo & Máximo & $\begin{array}{l}\text { Shapiro Wilk } \\
\text { p-valor }\end{array}$ \\
\hline \multirow{4}{*}{$\begin{array}{l}\text { Massa } \\
\text { corporal }\end{array}$} & \multirow{2}{*}{ GE } & pré-teste & 62,4017 & 1,28583 & 61,4 & 9,79258 & 37,1 & 87 & \multirow[t]{2}{*}{0,200} \\
\hline & & pós-teste & 61,4603 & 1,37572 & 60,05 & 10,47716 & 38,5 & 88,2 & \\
\hline & \multirow{2}{*}{ GC } & pré-teste & 60,5375 & 1,69859 & 62,05 & 10,74281 & 38,7 & 78 & \multirow[t]{2}{*}{0,116} \\
\hline & & pós-teste & 60,6875 & 1,69852 & 62,15 & 10,74237 & 38,7 & 78 & \\
\hline \multirow{4}{*}{ Estatura } & \multirow{2}{*}{ GE } & pré-teste & 1,5017 & 0,00817 & 1,51 & 0,06224 & 1,36 & 1,62 & \multirow[t]{2}{*}{0,028} \\
\hline & & pós-teste & 1,499 & 0,00818 & 1,505 & 0,06231 & 1,36 & 1,62 & \\
\hline & \multirow{2}{*}{ GC } & pré-teste & 1,4808 & 0,00922 & 1,49 & 0,05828 & 1,36 & 1,58 & \multirow[t]{2}{*}{0,194} \\
\hline & & pós-teste & 1,4808 & 0,00922 & 1,49 & 0,05828 & 1,36 & 1,58 & \\
\hline \multirow{4}{*}{ IMC } & \multirow{2}{*}{ GE } & pré-teste & 27,6059 & 0,47128 & 27,125 & 3,58919 & 19,78 & 36,21 & \multirow[t]{2}{*}{0,200} \\
\hline & & pós-teste & 26,8298 & 0,69753 & 27,06 & 5,31223 & 0 & 36,24 & \\
\hline & \multirow{2}{*}{ GC } & pré-teste & 27,625 & 0,75425 & 27,98 & 4,77027 & 17,92 & 35,2 & \multirow[t]{2}{*}{0,045} \\
\hline & & pós-teste & 27,6945 & 0,75575 & 28,13 & 4,7798 & 17,92 & 35,2 & \\
\hline
\end{tabular}

GE: grupo experimental; GC: grupo controle; IMC: índice de massa corpórea; Unidades de medida: massa corporal (Kg); Estatura (metros).

Na tabela 3, são apresentados os dados descritivos e a análise da normalidade da autonomia funcional (AF) do GE. As médias apresentadas em todos os testes de AF no pós-teste foram inferiores as encontradas no pré-teste. O IG - índice GDLAM apontou valores médios de 39,30 $+5,42$ no pré-teste e 35,17+4,73 no pós-teste. Embora o GE tenha apresentado melhor desempenho no pós-teste, estes foram classificados ${ }^{14}$ como "fraco" nos testes LPS $(x=13,34+2,29 s), \operatorname{LCLC}(x=61,64+7,41 s)$, VTC $(x=14,88+4,84$ s) e "regular" no LPDV $(x=4,36+1,37 s)$ e C10m $(x=6,95+1,36 s)$.

Tabela 3 - Análise descritiva e de normalidade da autonomia funcional do GE. Juazeiro do Norte, CE, 2009.

\begin{tabular}{|c|c|c|c|c|c|c|c|c|}
\hline & & Média & $\begin{array}{l}\text { Erro- } \\
\text { padrão }\end{array}$ & Mediana & $\begin{array}{l}\text { Desvio- } \\
\text { padrão }\end{array}$ & Mínimo & Máximo & $\begin{array}{l}\text { Shapiro } \\
\text { Wilk p- } \\
\text { valor }\end{array}$ \\
\hline \multirow[t]{2}{*}{$\mathrm{C} 10 \mathrm{~m}$} & pré-teste & 8,0766 & 0,21978 & 8,035 & 1,67382 & 5,56 & 13,78 & 0,200 \\
\hline & pós-teste & 6,9598 & 0,17885 & 6,515 & 1,36206 & 4,78 & 10,12 & \\
\hline \multirow[t]{2}{*}{ LPS } & pré-teste & 14,0522 & 0,37114 & 13,91 & 2,8265 & 8,28 & 22,1 & 0,164 \\
\hline & pós-teste & 13,3497 & 0,30193 & 13 & 2,29941 & 10 & 21,47 & \\
\hline \multirow[t]{2}{*}{ LCLC } & pré-teste & 67,9379 & 1,47419 & 67,42 & 11,22707 & 44,29 & 90,99 & 0,200 \\
\hline & pós-teste & 61,6431 & 0,97345 & 61 & 7,41357 & 48,36 & 84 & \\
\hline \multirow[t]{2}{*}{ VTC } & pré-teste & 17,4559 & 0,65886 & 16,775 & 5,01775 & 8,08 & 31,19 & 0,200 \\
\hline & pós-teste & 14,8455 & 0,63599 & 14,405 & 4,84356 & 3,05 & 32,43 & \\
\hline \multirow[t]{2}{*}{ LPDV } & pré-teste & 5,0474 & 0,20089 & 4,735 & 1,52992 & 3 & 11,76 & 0,000 \\
\hline & pós-teste & 4,3645 & 0,18095 & 4,105 & 1,37809 & 2,4 & 11,18 & \\
\hline \multirow[t]{2}{*}{ IG } & pré-teste & 39,3005 & 0,71223 & 38,885 & 5,4242 & 25,28 & 53,72 & 0,200 \\
\hline & pós-teste & 35,1705 & 0,62225 & 34,7613 & 4,73889 & 26,61 & 51,47 & \\
\hline
\end{tabular}

C10M - caminhar 10m; LPS - levantar-se da posição sentada; LPDV - Levantar-se da posição decúbito ventral; LCLC - Levantar-se da cadeira e locomover-se pela casa; VTC - Vestir e tirar uma camiseta; IG: Índice GDLAM. unidade de medida dos testes (segundos). 
A análise descritiva e a avaliação da normalidade da autonomia funcional do GC estão apresentadas na tabela 4. No que se refere às médias de desempenho, evidenciaram-se valores similares em todos os testes, tanto no pré quanto no pós-teste. A média do IG foi de 43,34+10,33 e 43,39+10,34 no pré e pós-teste, respectivamente. Este grupo apontou escores classificados ${ }^{14}$ como "fraco" em todos os testes, tanto na primeira quanto na segunda avaliação (pré e pós-teste).

Tabela 4 - Análise descritiva e de normalidade da autonomia funcional do GC. Juazeiro do Norte, CE, 2009.

\begin{tabular}{|c|c|c|c|c|c|c|c|c|}
\hline & & Média & $\begin{array}{l}\text { Erro- } \\
\text { padrão }\end{array}$ & Mediana & $\begin{array}{l}\text { Desvio- } \\
\text { padrão }\end{array}$ & Mínimo & Máximo & $\begin{array}{l}\text { Shapiro } \\
\text { Wilk p- } \\
\text { valor }\end{array}$ \\
\hline \multirow[t]{2}{*}{$\mathrm{C} 10 \mathrm{~m}$} & pré-teste & 8,9053 & 0,35176 & 8,67 & 2,22472 & 5,5 & 15,09 & 0,071 \\
\hline & pós-teste & 8,946 & 0,35345 & 8,725 & 2,23539 & 5,5 & 15,09 & \\
\hline \multirow[t]{2}{*}{ LPS } & pré-teste & 15,9578 & 0,94226 & 14,295 & 5,95936 & 5,68 & 30,12 & 0,042 \\
\hline & pós-teste & 15,9693 & 0,94527 & 14,24 & 5,9784 & 5,7 & 30,1 & \\
\hline \multirow[t]{2}{*}{ LCLC } & pré-teste & 73,1848 & 3,05859 & 72,275 & 19,34425 & 41,3 & 119,34 & 0,198 \\
\hline & pós-teste & 73,2175 & 3,05997 & 72,285 & 19,35294 & 41,31 & 119,35 & \\
\hline \multirow[t]{2}{*}{ VTC } & pré-teste & 18,983 & 0,90726 & 18,07 & 5,73804 & 8,52 & 32,4 & 0,146 \\
\hline & pós-teste & 18,9995 & 0,90683 & 18,065 & 5,73532 & 8,56 & 32,42 & \\
\hline \multirow[t]{2}{*}{ LPDV } & pré-teste & 6,253 & 0,47605 & 5,26 & 3,01083 & 2,56 & 13,09 & 0,003 \\
\hline & pós-teste & 6,276 & 0,47425 & 5,325 & 2,99941 & 2,58 & 13,1 & \\
\hline \multirow[t]{2}{*}{ IG } & pré-teste & 43,3457 & 1,63348 & 43,9625 & 10,33103 & 26,98 & 72,23 & 0,273 \\
\hline & pós-teste & 43,3998 & 1,63586 & 43,99 & 10,34612 & 27,01 & 72,25 & \\
\hline
\end{tabular}

C10M - caminhar 10m; LPS - levantar-se da posição sentada; LPDV - Levantar-se da posição decúbito ventral; LCLC - Levantar-se da cadeira e locomover-se pela casa; VTC - Vestir e tirar uma camiseta; IG: Índice GDLAM; unidade de medida dos testes (segundos).

$\mathrm{Na}$ comparação intragrupos, observou-se melhora significativa (redução no tempo de execução dos testes) em todos os testes do GDLAM e no IG no pós-teste do GE, a saber: C10m $(p=0,0001) ;$ LPS $(p=0,013) ;$ LCLC $(\mathrm{p}=0,0001) ; \operatorname{VTC}(\mathrm{p}=0,0001) ; \mathrm{LPDV}$ $(p=0,0001) ; \mathrm{IG}(p=0,0001)$. Já no $\mathrm{GC}$, houve piora significativa (aumento no tempo de execução dos testes) em todos os testes do GDLAM e no IG no pós-teste: $\mathrm{C} 10 \mathrm{~m}(\mathrm{p}=0,009)$; LPS $(p=0,012) ; \operatorname{LCLC}(p=0,034) ; \operatorname{VTC}(p=0,005) ;$ $\operatorname{LPDV}(p=0,002) ; \operatorname{IG}(p=0,0001)$ - figura 1 . 


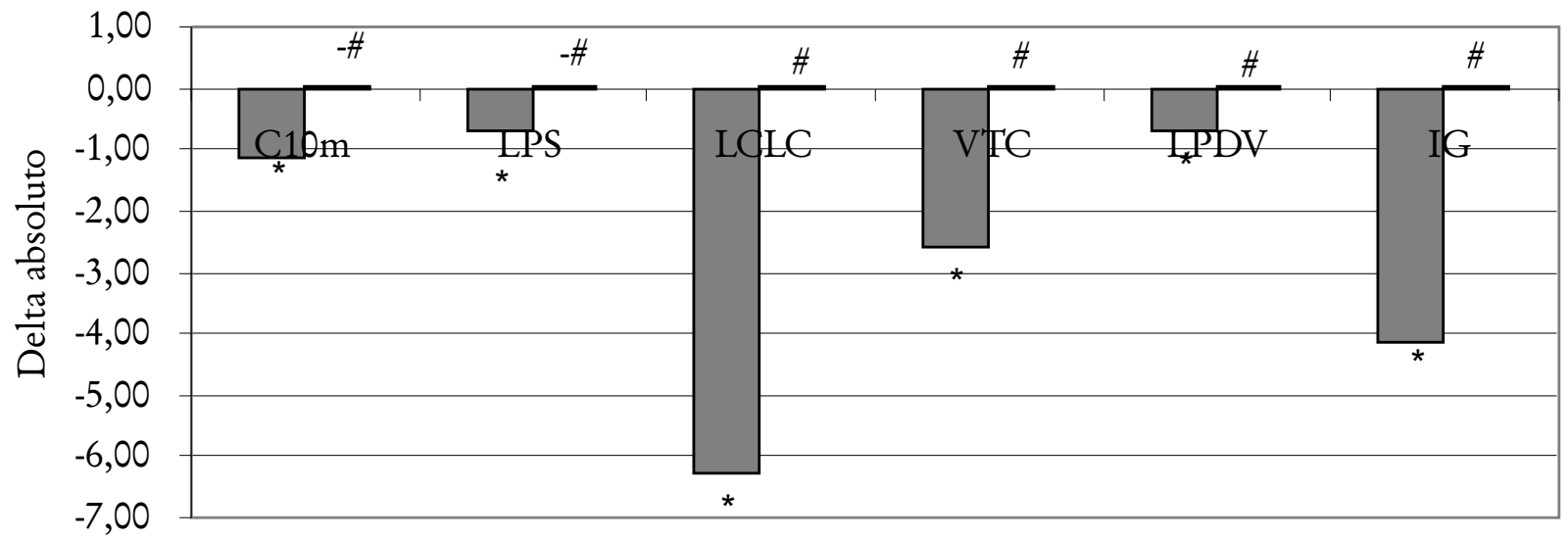

Testes do protocolo GDLAM

$\square \mathrm{GE} \quad \square \mathrm{GC}$

Figura 1- Comparação intragrupos dos níveis de autonomia funcional pelo delta absoluto (pós-testepré-teste).

C10M - caminhar 10m; LPS - levantar-se da posição sentada; LPDV - Levantar-se da posição decúbito ventral; LCLC - Levantar-se da cadeira e locomover-se pela casa; VTC - Vestir e tirar uma camiseta; IG: Índice GDLAM; unidade de medida dos testes (segundos).

A tabela 5 apresenta a comparação intergrupos. Nela, observa-se que o GE apresentou, no pós-teste, resultados significativamente satisfatórios $(\mathrm{p}<0,05)$ em todos os testes e no IG, quando comparados àqueles apresentados pelo GC.

Tabela 5 - Comparação intergrupos dos níveis de autonomia funcional pelo delta absoluto. Juazeiro do Norte, CE, 2009.

\begin{tabular}{lllrrrr}
\hline & & & \multicolumn{2}{c}{$\begin{array}{c}\text { Delta } \\
\text { Absoluto }\end{array}$} & p-valor & Intervalo de Confiança \\
\hline \multirow{2}{*}{ C10m } & pré-testeGE & pré-testeGC & $-0,83$ & 0,193 & $-1,898$ & 0,241 \\
\multirow{2}{*}{ LPS } & pós-testeGE & pós-testeGC & $-1,99$ & $\mathbf{0 , 0 0 0}$ & $-3,056$ & $-0,917$ \\
& pré-testeGE & pré-testeGC & $-1,91$ & 0,202 & $-4,393$ & 0,582 \\
\multirow{2}{*}{ LCLC } & pós-testeGE & pós-testeGC & $-2,62$ & $\mathbf{0 , 0 3 4}$ & $-5,107$ & $-0,132$ \\
& pré-testeGE & pré-testeGC & $-5,25$ & $\mathbf{0 , 3 6 9}$ & $-13,563$ & 3,070 \\
\multirow{2}{*}{ VTC } & pós-testeGE & pós-testeGC & $-11,57$ & $\mathbf{0 , 0 0 2}$ & $-19,891$ & $-3,258$ \\
& pré-testeGE & pré-testeGC & $-1,53$ & 0,577 & $-4,584$ & 1,530 \\
\multirow{2}{*}{ LPDV } & pós-testeGE & pós-testeGC & $-4,15$ & $\mathbf{0 , 0 0 3}$ & $-7,211$ & $-1,097$ \\
& pré-testeGE & pré-testeGC & $-1,21$ & $\mathbf{0 , 0 7 6}$ & $-2,492$ & 0,081 \\
\multirow{2}{*}{ IG } & pós-testeGE & pós-testeGC & $-1,91$ & $\mathbf{0 , 0 0 1}$ & $-3,198$ & $-0,625$ \\
& pré-testeGE & pré-testeGC & $-4,05$ & $\mathbf{0 , 0 9 0}$ & $-8,491$ & 0,401 \\
& pós-testeGE & pós-testeGC & $-8,23$ & $\mathbf{0 , 0 0 0}$ & $-12,675$ & $-3,783$ \\
\hline
\end{tabular}

C10M - caminhar 10m; LPS - levantar-se da posição sentada; LPDV - Levantar-se da posição decúbito ventral; LCLC - Levantar-se da cadeira e locomover-se pela casa; VTC - Vestir e tirar uma camiseta; IG: Índice GDLAM; unidade de medida dos testes (segundos) 


\section{DISCUSSÃO}

Os valores de IMC (tabela 2) indicaram que ambos os grupos estavam classificados como sobrepeso, resultado semelhante ao apresentado por Vale et al. ${ }^{13}$ em estudo realizado com idosos participantes de grupos de terceira idade. Também o estudo de Cader et al. ${ }^{17}$ avaliando a idosas sedentárias, asiladas e praticantes de hidroginástica, encontrou o IMC classificado como sobrepeso em todos os grupos. Contudo, no presente estudo, as idosas que participaram do grupo experimental apresentaram diminuição nos valores médios de massa corporal e, consequentemente, no IMC, mas não o suficiente para atingir níveis considerados normais para o estado nutricional, de acordo com a classificação adotada.

Em relação à autonomia funcional, as idosas apresentaram melhor desempenho em todos os testes na avaliação pós-intervenção. Resultados próximos foram encontrados por Guimarães et al. ${ }^{18}$ segundo os quais idosas submetidas a um programa de atividade física que incluía a realização de caminhadas, com frequência semanal de três vezes e duração de $60 \mathrm{~min}$., por pelo menos três meses, apresentaram diminuição no tempo de execução em todos os testes da bateria GDLAM, e consequentemente no IG, o que reflete a melhora no nível de autonomia funcional. Resultados semelhantes também são observados nos estudos de Varejão et al., ${ }^{19}$ quando submeteram um grupo de idosas a um programa de intervenção de baixo impacto, composto por aulas de alongamento, explicitando que embora o programa de atividades físicas tenha sido diferenciado da presente pesquisa, seus resultados também foram positivos.

Em estudo realizado por Vale et al. ${ }^{20} \mathrm{com}$ idosos submetidos a um treinamento de força, foi verificado que depois de realizado o tratamento experimental, o grupo submetido à intervenção reduziu os tempos marcados nos testes de autonomia funcional C10M, LPS e LPDV. Quando comparado com o grupo controle, o grupo experimental realizou todos os testes em menor tempo. No estudo ora realizado, os tempos de execução nos testes C10m, LPS e
LPDV também tenderam a diminuir na avaliação pós-intervenção e apresentaram melhores resultados em relação ao grupo controle.

Varejão et al. ${ }^{21}$ realizaram intervenção com atividades especificas de alongamento em um período de 24 semanas, e obtiveram efeitos positivos e significativos na autonomia funcional de idosos, apresentando resultados melhores do que os encontrados no presente estudo. Aragão et al.,22 estudando idosas regularmente ativas $\mathrm{da}$ cidade de Rio de Janeiro, identificaram valores menores que o GE-Pré, porém, maiores que o GE-Pós do presente estudo, com média de $7,28+1,79$ segundos. Corroborando ainda os resultados do teste $\mathrm{C} 10 \mathrm{M}$ da presente pesquisa, Guimarães et al. ${ }^{18}$ encontraram resultados semelhantes tanto no GE-pré como no GE-Pós, apresentando valores médios respectivos de 8,37 e 6,29 segundos.

Belloni et al..$^{23}$ avaliaram a autonomia funcional de idosas praticantes e não-praticantes de hidroginástica e perceberam melhor desempenho para o primeiro grupo. Neste, o desempenho foi considerado como "muito bom" nos testes de C10M, LPS, LPDV e "bom" no teste LCLC. Em relação ao presente estudo, embora o GE tenha apontado melhoras no desempenho dos testes $\mathrm{AF}$ pós-intervenção, estes foram classificados, ${ }^{14}$ como "fraco" nos testes LPS, LCLC, VTC e "regular" no LPDV e C10M.

Silva et $\mathrm{a}^{24}$ verificaram o efeito de um treinamento de força muscular com duração de 20 semanas sobre o nível de autonomia funcional de idosas residentes na cidade do Rio de Janeiro e perceberam que o grupo experimental apresentou melhor desempenho no pós-teste, quando comparado ao grupo controle. O grupo experimental, no estudo citado, apresentou valores médios de IG de 21,70, enquanto que no presente estudo, o valor médio desta variável para o GE foi de 35,17 .

Estudo desenvolvido por Coelho \& Coelho ${ }^{6}$ com idosas do estado do Piauí, submetidas a programas de caminhadas e hidroginástica composto por três seções semanais durante um 
período de 12 semanas, apresentou resultados finais no teste de VTC de 9,1+2,6 e 13,7+3,2, respectivamente. Esses resultados, embora melhores do que os obtidos no presente estudo, não refletem a melhoria real no desempenho desta tarefa, visto que as participantes da intervenção, no estudo citado, apresentaram melhoria média de 1,1 e 0,6 segundos.

Pode-se ainda deduzir, a partir dos três experimentos exemplificados, que atividades ao ar livre envolvendo movimentos contra a resistência gravitacional (caminhada), ao contrário da resistência da água (hidroginástica), parecem apresentar resultados mais satisfatórios na melhoria da autonomia nesta atividade.

Efeitos da intervenção com caminhada e hidroginástica, em pesquisa já apresentada nesta discussão, demonstraram que após programa de treinamento, os resultados obtidos no teste de LPDV foram, em média, $3,7 \pm 2,1$ e 4,7 $\pm 4,1$ segundos, respectivamente, intervindo de forma expressiva no desempenho inicial das participantes, que foi de 7,6 $\pm 3,0$ segundos na caminhada e 5,6 $6 \pm 4,2$ segundos na hidroginástica. ${ }^{5}$ Esses resultados foram numericamente melhores do que os desta pesquisa, mesmo sendo realizada em menor intervalo de tempo, parecendo ter surtido melhor efeito do que a presente pesquisa.

Os resultados do IG para o GE-Pós foi de $27,8 \pm 4,8$ e do GC de $33,7 \pm 4,8$, nos quais se pode observar diferença significativa $(p=0,00)$ na melhoria dos resultados, após intervenção tanto em relação ao GC quanto o início do GE-Pré, que era de $30,8 \pm 5,1$. Guimarães et al. ${ }^{18}$ verificaram efeitos similares em grupo de idosas após intervenção com programa de atividade física, alterando seus resultados de $38,6 \pm 4,3$ para $27,3 \pm 2,02$ segundos.

Estes resultados de IG se tornam muito importantes, pois resultam da interação dos testes isolados do GDLAM, os quais refletem a capacidade funcional dos idosos e sua autonomia, influenciando diretamente em sua qualidade de vida. Comparando com os valores sugeridos pelo GDLAM, o grupo que realizou intervenção, apesar de reduzir seu IG de forma significativa, continuou classificado como fraco, resultado inferior aos apresentados por Guimarães et al. ${ }^{18}$ e Pereira et al., ${ }^{25}$ este último um dos precursores da utilização deste instrumento no Brasil para amostras idosas.

Para Cunha et al., ${ }^{26}$ esta melhora no desempenho do idoso será considerada saudável no indivíduo que, chegando aos 80 anos de idade, seja capaz de administrar sua própria vida e determinar quando, onde e como se darão suas atividades de lazer, convívio social e trabalho, condição esta em que a média do grupo estudado se aproxima.

\section{CONCLUSÕES}

O estudo mostra que o grupo estudado, depois de submetido às atividades de caminhada, obteve resultados superiores e significativos aos iniciais e ao grupo controle.

Estes resultados contribuem de forma relevante para essa população, pois por menor que seja a melhora conquistada na condição física e de autonomia nesta faixa etária, repercutirá em menor dificuldade para a realização das atividades da vida diária, melhorando assim sua capacidade funcional, tornando o idoso menos dependente no contexto social em que vive.

Alguns fatores podem ter contribuído para o pouco nível de melhora dos idosos, como o baixo condicionamento físico inicial e fatores externos não controlados. Justifica-se, desta forma, a realização de novos estudos com esta população, para que fique mais clara a influência da caminhada, controlando-se variáveis que possam ter influenciado os resultados. 


\section{REFERENNCIAS}

1. IBGE.Instituto Brasileiro de Geografia e Estatística. População Idosa. Disponível em < http://www.ibge.gov.br. Acesso em 18 jun.2008.

2. Audrey SA, Débora FO, Marcio RB, Estélio HMD. Os efeitos da prática do yoga sobre a capacidade física e autonomia funcional em idosas. Fitness \& performance journal : Órgão oficial do Colégio Brasileiro de Atividade Física, Saúde e Esporte 2006; 4:243-9.

3. Berlezi EM, Rosa PV, Souza ACA, Schneider RH. Comparação antropométrica e do nível de aptidão física de mulheres acima de 60 anos praticantes de atividade física regular e não praticantes. Revista brasileira de geriatria e gerontologia 2006; 9(3).

4. Matsudo SM, Matsudo VKR, Barros Neto TL, Araújo TL. Evolução do perfil neuromotor e capacidade funcional de mulheres fisicamente ativas de acordo com a idade cronológica.

Revista brasileira de medicina do esporte 2006; 9(6):365-76.

5. Farinatti PTV, Oliveira RB, Pinto VLM, Monteiro WD, Francischetti E. Programa domiciliar de exercícios: efeitos de curto prazo sobre a aptidão física e pressão arterial de indivíduos hipertensos. Arq Bras Cardiol 2005;84(6):473-9.

6. Coelho CSE, Coelho IC. Comparação dos benefícos obtidos através da caminhada e da hidroginástica para a terceira idade. < acesso em 05 nov 2008>. ANAIS do II Encontro de Educação Física e Áreas Afins. Dispon[ivel em :http://www.ufpi.br/def/arquivos/file/ COMPARAO\%20DOS\%20BENEFCOS\%20OBTI DOS\%20ATRAVS\%20DA\%2OCAMINHADA\%2OE\% $200 A \% 2$ OHIDROGINSTICA\%20PARA\%20A\%2 OTERCEIRA\%2OIDADE.pdf.

7. WMA - World Medical Association. Declaration of Helsinki. Ethical Principles for Medical Research Involving Human Subjects. 59th WMA General Assembly, Seoul, October 2008.

8. Dantas EHM, Vale RGS. Protocolo GDLAM de avaliação da autonomia. Fitness \& performance journal : Órgão oficial do Colégio Brasileiro de Atividade Física, Saúde e Esporte 2004;3(3):169-80.

9. Sipilä S, Multanen J, Kallinen M, Era P, Suominen H. Effects of strength and endurance training on isometric muscle strength and walking speed in elderly women. Acta Physiol Scand 1996;156: 457-64.
10. Guralnik JM, Ferrucci L, Pieper CF, Leveille SG, Markides KS, Ostir GV, Studenski S, Berkman LF, Wallace RB. Lower extremity function and subsequent disability consistency across studies, predictive models and value of gait speed alone compared with the short physical performance battery. J Gerontol 2000; 55(4): 221-31.

11. Alexander NB, Ulbrich J, Raheja A, Channer D. Rising from the floors in older adults. J Am Geriatr Soc 1997;45(5):564-9.

12. Andreotti RA, Okuma SS. Validação de uma bateria de testes de atividades da vida diária para idosos fisicamente independentes. Revista brasileira de educação física e esporte 1999;13(1):46-66.

13. Vale RGS, Pernambuco CS, Novaes JS, Dantas EHM. Teste de autonomia funcional: vestir e tirar uma camiseta (VTC). Revista brasileira de ciencia \& movimento 2006;14(3):71-8.

14. Vale RGS. Avaliação da autonomia funcional do idoso. Fitness \& performance journal : Órgão oficial do Colégio Brasileiro de Atividade Física, Saúde e Esporte 2005; 4(1):4.

15. American College of Sports Medicine. Diretrizes do ACSM para os testes de esforço e sua prescrição. 6.ed. Rio de Janeiro: Guanabara Koogan; 2003.

16. V Diretrizes Brasileiras de Hipertensão Arterial. Arquivo Brasileiro de Cardiologia. São Paulo: Set. 2007; 89(3). < Acesso em 07 nov.de 2008 > Disponível em: http://www.scielo.br/ scielo.

17. Cader SA, Vale RGD, Pereira FF, Dantas EHM. Comparação da Pimáx e da qualidade de vida entre idosas sedentárias, asiladas e praticantes de hidroginástica. Fitness \& performance journal : Órgão oficial do Colégio Brasileiro de Atividade Física, Saúde e Esporte 2006 ; 5(2) : 101-8.

18. Guimarães AC, Rocha CAQC, Gomes ALM, Cader SA, Dantas EHM. Efeitos de um programa de atividade física sobre o nível de autonomia de idosos participantes do programa de saúde da família. Fitness \& performance journal : Órgão oficial do Colégio Brasileiro de Atividade Física, Saúde e Esporte 2008;7(1):5-9.

19. Varejão RV, Melo R, Barros R, Vale RGS, Aragão JCB, Amorim FS, Dantas EHM. Comparação dos efeitos do alongamento e do flexionamento ambos passivos sobre os níveis de flexibilidade, 
autonomia e qualidade de vida do idoso. Revista brasileira de ciencia \& movimento 2007; 15(2): 87-95

20. Vale RGS, Barreto ACG, Novaes JS, Dantas EHM. Efeitos do treinamento resistido na força máxima, na flexibilidade e na autonomia funcional de mulheres idosas. Revista brasileira de cineantropometria $\&$ desempenho humano 2006;8(4) p.52-8.

21. Varejão RV, Dantas EHM, Matsudo SMM. Comparação dos efeitos do alongamento e do flexionamento, ambos passivos, sobre os níveis de flexibilidade, capacidade funcional e qualidade de vida do idoso. Universidade Castelo Branco. Revista brasileira de ciencia \& movimento 2007;15(2):87-95.

22. Aragão JCB. Efeitos da resistência muscular localizada visando a autonomia e a qualidade de vida de idosos idosos. (dissertação) Rio de Janeiro: Universidade Castelo Branco; 2002. 332 f.
23. Belloni D, Albuquerque AC, Rodrigues TO, Mazini Filho ML, Silva VF. Estudo comparativo entre a autonomia funcional de mulheres idosas praticantes e não praticantes de hidroginástica. Revista de Educação Física 2008 mar;140:20 -25

24. Silva JGFB, Cader SA, Dopico X, Soler EO, Dantas EHM. Fortalecimiento muscular, nivel de fuerza muscular y autonomia funcional en una población de mujeres mayores. Revista espanhola de geriatria y gerontologia 2009; 44(5):256-61.

25. Pereira FF, Monteiro N, Portal MND, Vale RGS, Dantas EHM. Perfil de um grupo de mulheres idosas residentes no condomínio Rio 2, no município do Rio de Janeiro. Fitness \& performance journal : Órgão oficial do Colégio Brasileiro de Atividade Física, Saúde e Esporte 2005;4(6):352-6.

26. Cunha RCL, et al. Análise da flexibilidade e da autonomia funcional em idosos não praticantes de exercício físico. Livro de Memória do III Congresso Cientifico Norte-Nordeste. Fortaleza, 2007. 
